\title{
Decision-Making of an Integrated Supply Chain with Hybrid Sales Channels to Cope with Dual Cost Disruptions
}

\author{
Yuyan WANG \\ School of Management Science and Engineering, Shandong University of Finance and Economics, \\ Jinan 250014, China \\ E-mail: wangyuyan1224@126.com \\ Yuanyuan ZHANG \\ School of Management Science and Engineering, Shandong University of Finance and Economics, \\ Jinan 250014, China
}

\begin{abstract}
For an integrated supply chain with an online direct channel and a traditional retail channel competing with each other, solutions are be identified as to the two channels' ordering policies, product pricing strategies, and optimal product output, when product costs in the two channels are disrupted in different ways. Findings are as follows: 1) For the integrated supply chain, when unanticipated events lead to product cost increase, market size will shrink, and the system profit is harmed. In contrast, when unanticipated events lead to reduced product cost, market size will expand, and system profit increases; 2) Production strategies applicable to normal situations have certain robustness, and should be maintained when product cost disruption caused by unanticipated events is relatively small; 3) When product cost disruption caused by unanticipated events is relatively large, product sales price should be first adjusted, and aligned with the way that product cost is disrupted. Meanwhile, order quantity and product output should also be properly adjusted. That is, order quantity and output need to be reduced when product cost increases; order quantity and output need to be increased when product cost is reduced. In the end, this paper employs numerical examples to testify the findings. Research conclusions help to further enrich and extend the theoretic basis of "supply chain disruption management", and are helpful for researchers' further study.
\end{abstract}

Keywords integrated supply chain; hybrid sales channel; disruption

\section{Introduction}

Modern supply chain management increasingly focuses on lean management, with a purpose of improving supply chain efficiency and reducing cost. Lean supply chain, however, is extremely vulnerable when confronted with various unanticipated events like natural disasters, foreign exchange rate fluctuation, public health events etc. Unanticipated events bring about various consequences, like temporary disruption of raw material supply, huge demand fluctuation (for instance, the shortage of goods and medicines to prevent SARS (Severe Acute

Received May 14, 2015, accepted August 12, 2015

Supported by the National Natural Science Foundation of China (71501111), Natural Science Foundation of Shandong Province (ZR2014JL046) 
Respiratory Syndrome) during its breakout in China), traffic facility unavailability or interruption (US air transport after the 911), unavailability of plants, warehouses, and offices (plants producing computer motherboard in Taiwan after the 921 Taiwan earthquake), direct damage to goods or services (the impact of nuclear leakage on food in Japan earthquake in March 2011), communication channel jam and so on. Consequently, huge losses are incurred. For example, Hendricks and Singhal ${ }^{[1]}$ found that, in the short term, supply chain disruptions caused by unanticipated events resulted in a $10.28 \%$ loss of corporate fortune; in the long run, the breakout of unforeseen events in a supply chain led to $33 \% \sim 40 \%$ reduction of corporate normal average revenue ${ }^{[2]}$. Therefore, huge direct and indirect losses of companies and supply chains caused by unanticipated events require better supply chain disruption management ${ }^{1}$.

\section{Literature Review}

In recent years, the frequency and intensity of various unanticipated events have continuously increased. It has become an urgent problem for Supply chain members to deal with disruptions caused by unanticipated events and this issue attracted extensive academic attention ${ }^{[5-6]}$. Up to now, studies on supply chain disruption management are concentrated on the following aspects:

1) Studies on decision-making as to coping with sudden change of market demand. When unanticipated events break out, market demand changes differently in different sales channels, and the corresponding adjustment strategies vary. As a start, $\mathrm{Xu}$, et al. ${ }^{[7]}$ proposed supply chain coordination under disrupted demand when there was a linear relationship between price and demand; afterwards, Qi, et al. ${ }^{[8]}$ explored coordination mechanism for a supply chain to handle unanticipated events, and analyzed how a supply chain of one manufacturer and one retailer used quantity discount contract to handle unanticipated events under linear demand. On this basis, Xiao, et al. ${ }^{[9]}$ extended supply chain coordination issue under unanticipated events to a two-echelon supply chain of one manufacturer and two retailers. Chen ${ }^{[10]}$ studied a supply chain of one supplier and many retailers, which dominated by retailers, and analyzed its decision problem when unanticipated events happen. Furthermore, Huang ${ }^{[11]}$ extended these studies to dual-channel supply chain system, and constructed a dual-channel supply chain model of one manufacturer and one retailer. The study also analyzed optimal production and pricing strategies for the integrated supply chain system composed of the two parties when there was a sudden change in demand, and indicated that these optimal strategies had certain robustness.

2) Studies in decision making on how to deal with sudden product supply change or cost change. For example, Xu, et al. ${ }^{[12]}$ constructed a supply chain of one manufacturer and one retailer, and analyzed the coordination of supply chain with quantity discount contract when production cost was disrupted; on this basis, $\mathrm{Li}^{[13]}$ established a supply chain model of one retailer and two competing suppliers, and obtained suppliers' optimal equilibrium price when unanticipated events resulted in supply fluctuation, and provided production strategies to maximize system interest. In addition, Wilson ${ }^{[14]}$ used system dynamics to study the impact of

\footnotetext{
${ }^{1}$ Causen, et al. (see [3]) first put forward the concept of disruption management. He thinks that disruption refers to situations that some key resources, like staff or equipment, deviate from their intended normal conditions and plan, so they must be handled and adjusted. On this basis, the theory of supply chain disruption management is formed (see [4]).
} 
unanticipated transportation events on production efficiency of a supply chain, and demonstrated that the influence of unforeseen events could be greatly weakened under VMI model.

3) Decision studies on how to handle various consequences of unanticipated events. This has been the difficult part of current studies, and only limited results have been achieved. Xiao ${ }^{[15]}$ discussed using quantity discount contract to coordinate a supply chain of many competing retailers, and extended the model to scenarios that production cost and market demand were both disrupted. Afterwards, Davarzani ${ }^{[16]}$ formulated a supply chain model of two suppliers, and researched coordination strategies of supply chain members when they are confronted with supply change or demand fluctuation caused by unanticipated events; corresponding management strategies are proposed to deal with the various consequences caused by unanticipated events, and auto manufacturing supply chain adjustment strategy was taken as a case.

4) Research on strategies to deal with unanticipated events. Hallikas ${ }^{[17]}$ analyzed major influence factors of supply chain risks, and identified all the potential risks in a supply chain. Tomlin ${ }^{[18]}$ studied unanticipated events management strategies from different perspectives, and put forward procurement strategies, inventory strategies, and emergent line change strategies to tackle unanticipated events. On this basis, Schmitt ${ }^{[19]}$ constructed a model of multi-echelon supply chain that handles unanticipated events, and proposed the optimal strategies to maintain customer service level in unanticipated events. And findings showed that previous inventory and product recycle strategies both affected system restoration after unanticipated events.

Moreover, researchers have made some achievements in other aspects. For example, Craighe$\mathrm{ad}^{[20]}$ raised six propositions reflecting the severity of supply chain unanticipated events by analyzing supply chain structure; Dowty ${ }^{[21]}$ discussed organization culture in supply chain disruption management; Zegordi, et al ${ }^{[22]}$ employed colored Petri-nets to construct a supply chain analysis model to cope with unanticipated events, and demonstrated the evolution process of unanticipated events.

To sum up, studies on supply chain disruption management have born many fruits. From various perspectives, researchers analyzed strategies to handle unanticipated events. But few existing achievements aimed at complicated conditions. In particular, systematic studies are insufficient when unanticipated events caused disruptions in various aspects. Furthermore, current studies mainly focused on supply chain with single sales channel, but there are more supply chains with mixed sales channel existing in practice. And in this kind of supply chain system, competition exists between different sales channels. Here a question comes: are these previous studies applicable for supply chains with hybrid sales models? And this is the question to be solved.

On this basis, this paper studies an integrated supply chain with hybrid sales channels competing with each other; and analyzes production adjustment strategies of an integrated supply chain to cope with unanticipated events, when sales channels compete with each other and production cost disruption conditions vary in the two sales channels. The purpose is to further enrich supply chain adjustment strategy system to deal with unanticipated events. 


\section{Models and Production Strategies in Normal Situations}

An integrated supply chain refers to a supply chain system that all members form strategic alliances via valid contracts, and all parties make decisions to maximize the systematic profit of the supply chain, and collectively formulate strategies in terms of product sales price, order quantity, and so on (see [11]). Throughout an integrated supply chain, information is symmetric, i.e., each member knows the cost structure, and profit function, etc. of his own and others (see [23]).

An integrated supply chain system of one manufacturer and one retailer is studied in this paper. Two sales channels exist in the system, i.e., traditional retail channel where manufacturer produces and sells to retailers who then sell to the market, and online direct sales channel where manufacturer not only produces, but also sells directly to consumers who place orders online with the help of network information technology. Thus, this constitutes a supply chain with hybrid sales channel. Structure of an integrated supply chain system is shown in Figure 1.

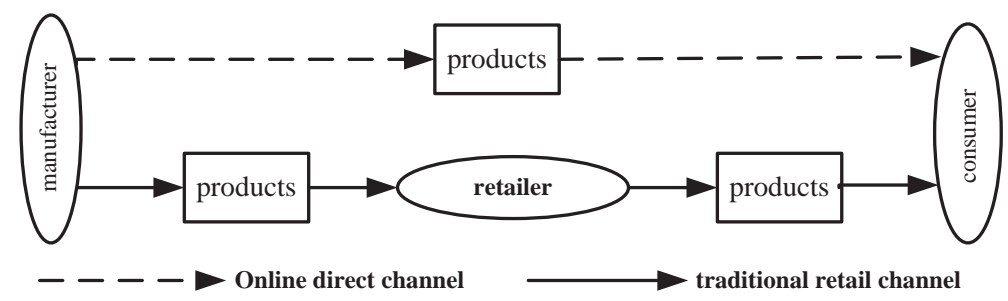

Figure 1 Structure of an integrated supply chain system

In an integrated supply chain, products sold through the two channels are identical. But sales costs of these two sales channels are different, so identical products are sold at different prices through these two channels. Consequently, there is competition between these two sales channels.

Assume sales price in traditional retail channel is $p_{i}$; sales price in direct sales channel is $p_{d}$. Given that competition exists between two sales channels and sales price is a major influence factor of sales volume, in view of the hypothesis forms of Atalay ${ }^{[24]}$ and Ferrer ${ }^{[25]}$, assume that sales function is as follows, when products are sold through retail channel:

$$
q_{i}=\alpha_{i}-p_{i}+\beta p_{d}
$$

And when products are sold in the direct channel, the sales function is

$$
q_{d}=\alpha_{d}-p_{d}+\beta p_{i}
$$

where $\alpha_{i}$ and $\alpha_{d}$ represent the maximum market size faced by retail channel and direct channel respectively, and consumers generally prefer to buy from retailers, due to traditional buying customs. Therefore traditional retail channel dominates and direct channel is supplementary in these two sales channels. Correspondingly, retail channel market size is bigger than direct channel market size, i.e., assume $\alpha_{i}>\alpha_{d} ; \beta(0<\beta<1)$ demonstrates the competition degree between the two sales channels; the larger $\beta$ is, the fiercer channel competition is, and the channel's sales is more disrupted and influenced by the other channel. 
Production cost is identical for the products sold in both channels, but sales cost varies in the two channels. Assume product cost (both production cost and sales cost are included) is $c_{i}$ when products are sold through retail channel, and product cost (both production cost and sales cost are included) is $c_{d}$ when products are sold through direct channel. Compared with retail channel, direct channel saves cost in transportation, inventory, advertisement, etc. by streamlining some operation procedures, and product cost in direct channel is lower than that in retail cost, i.e., assume $0<c_{d}<c_{i}$.

Based on the above assumptions and notation explanations, profit function $\pi$ of an integrated supply chain can be obtained as follows:

$$
\pi=\left(p_{i}-c_{i}\right) q_{i}+\left(p_{d}-c_{d}\right) q_{d}
$$

In normal situations, the retailer and the manufacturer determine related sales price and order quantity in order to schedule production process, with an aim of maximizing supply chain system profit.

Combining Formulaes (1) and (2), and based on the one-order optimality condition of Formula (3), then we obtain

Conclusion 1 In normal situations, the optimal profit of an integrated supply chain is

$$
\pi^{*}=\frac{\left[\alpha_{i}+\beta \alpha_{d}-\left(1-\beta^{2}\right) c_{i}\right]\left(\alpha_{i}-c_{i}+\beta c_{d}\right)}{4\left(1-\beta^{2}\right)}+\frac{\left[\alpha_{d}+\beta \alpha_{i}-\left(1-\beta^{2}\right) c_{d}\right]\left(\alpha_{d}-c_{d}+\beta c_{i}\right)}{4\left(1-\beta^{2}\right)} .
$$

Here, the optimal sales price of products sold through retail channel is

$$
p_{i}^{*}=\frac{\alpha_{i}+\beta \alpha_{d}+\left(1-\beta^{2}\right) c_{i}}{2\left(1-\beta^{2}\right)}
$$

The optimal sales price of products sold through direct channel is

$$
p_{d}^{*}=\frac{\alpha_{d}+\beta \alpha_{i}+\left(1-\beta^{2}\right) c_{d}}{2\left(1-\beta^{2}\right)} .
$$

And there is $p_{i}^{*}>p_{d}^{*}$, i.e., sales price of products sold through traditional retail channel is higher than that sold through direct sales channel.

Accordingly, manufacturer's optimal output is

$$
q^{*}=\frac{\alpha_{i}-c_{i}+\beta c_{d}}{2}+\frac{\alpha_{d}-c_{d}+\beta c_{i}}{2},
$$

of which, retailer's optimal order quantity is

$$
q_{i}^{*}=\frac{\alpha_{i}-c_{i}+\beta c_{d}}{2} .
$$

The optimal order quantity of direct channel is

$$
q_{d}^{*}=\frac{\alpha_{d}-c_{d}+\beta c_{i}}{2} .
$$

Conclusion 1 has following implications: In normal situations, product sales prices in both channels are affected by their market sizes, product cost, as well as their competition; the fiercer their competition is, the higher sales prices in both channels are. Order quantities of both channels are influenced by product market size, product cost and channel competition, and the order quantities of both channels increase as competition condition $\beta$ intensified. 


\section{Models and Adjustment Strategies Under Product Cost Disrup- tions in Both Channels}

For a supply chain in normal situations, the optimal output $q^{*}$ is obtained by the manufacturer according to market demand forecast of hybrid sales channels and production is scheduled. If unanticipated events break out before the sales season, they will lead to changes in product cost, i.e., cost of products sold through retail channel has changed from $c_{i}$ to $c_{i}+\Delta c_{i}$; cost of products sold through direct channel has changed from $c_{d}$ to $c_{d}+\Delta c_{d}$, where $c_{i}+\Delta c_{i}>0, c_{d}+\Delta c_{d}>0$.

Changes of product cost then result in changes of sales in both channels after the breakout of unanticipated events. Assume that sales volume in retail channel is $\bar{q}_{i}$, and that in direct channel is $\bar{q}_{d}$ after the breakout of unanticipated events. Assume changes of order quantity in the two channels are $\Delta q_{i}=\bar{q}_{i}-q_{i}^{*}$ and $\Delta q_{d}=\bar{q}_{d}-q_{d}^{*}$, respectively. As a result, order quantity changes incurred by unforeseen events result in corresponding cost change. If $\Delta q_{i}>0$ and $\Delta q_{d}>0$, increased product quantities $\Delta q_{i}$ and $\Delta q_{d}$ need extra cost of $\lambda_{i+}\left(0<\lambda_{i+}<c_{i}\right)$ and $\lambda_{d+}\left(0<\lambda_{d+}<c_{d}\right)$ if $\Delta q_{i}<0$ and $\Delta q_{d}<0$, surplus product quantities $-\Delta q_{i}$ and $-\Delta q_{d}$ lead to extra disposal cost of $\lambda_{i-}\left(0<\lambda_{i-}<c_{i}\right)$ and $\lambda_{d-}\left(0<\lambda_{d-}<c_{d}\right)$. Meanwhile, assume product sales prices will be accordingly adjusted too, after unforeseen events break out. And adjusted product sales price in retail channel is $\bar{p}_{i}$, and that in direct channel is $\bar{p}_{d}$.

So, with the outbreak of unforeseen events, profit function of an integrated supply chain has become

$$
\begin{aligned}
\bar{\pi}= & \left(\bar{p}_{i}-c_{i}-\Delta c_{i}\right) \bar{q}_{i}+\left(\bar{p}_{d}-c_{d}-\Delta c_{d}\right) \bar{q}_{d} \\
& -\left[\lambda_{i+}\left(\bar{q}_{i}-q_{i}^{*}\right)^{+}+\lambda_{d+}\left(\bar{q}_{d}-q_{d}^{*}\right)^{+}+\lambda_{i-}\left(q_{i}^{*}-\bar{q}_{i}\right)^{+}+\lambda_{d-}\left(q_{d}^{*}-\bar{q}_{d}\right)^{+}\right],
\end{aligned}
$$

where $\lambda_{i+}\left(\bar{q}_{i}-q_{i}^{*}\right)^{+}$and $\lambda_{d+}\left(\bar{q}_{d}-q_{d}^{*}\right)^{+}$respectively represent the extra costs of supply chain system incurred by increased production; $\lambda_{i-}\left(q_{i}^{*}-\bar{q}_{i}\right)^{+}$and $\lambda_{d-}\left(q_{d}^{*}-\bar{q}_{d}\right)^{+}$respectively represent the extra disposal costs of supply chain system incurred by surplus products. It can be obtained

from Formula (10) that $A=\frac{\partial^{2} \bar{\pi}}{\partial^{2} q_{i}^{2}}=-\frac{2}{1-\beta^{2}}, B=\frac{\partial^{2} \bar{\pi}}{\partial q_{i} \partial q_{d}}=-\frac{2 \beta}{1-\beta^{2}}, C=\frac{\partial^{2} \bar{\pi}}{\partial^{2} q_{d}^{2}}=-\frac{2}{1-\beta^{2}}$. Given that $A<0, A C-B^{2}=\frac{4}{1-\beta^{2}}>0$, Hessian matrix $\left[\begin{array}{cc}A & B \\ B & C\end{array}\right]$ of Formula (10) is negative definite; Formula (10) is a strictly concave function, and its unique optimal solution exists.

Next, supply chain strategies after cost disruption are analyzed, and assume the optimal order quantity in retail channel and direct channel are $\bar{q}_{i}^{*}$ and $\bar{q}_{d}^{*}$, respectively. By comparing the optimal order quantities before and after cost disruption, it is obtained that,

Conclusion 2 After unforeseen events break out, and when costs are disrupted in an integrated supply chain, there are

1) when $\Delta c_{i}>0, \Delta c_{d}>0$, the following scenarios will not happen to the optimal order quantities in either the retail channel or the direct channel:

(a) $\bar{q}_{i}^{*} \geq q_{i}^{*}$ and $\bar{q}_{d}^{*}>q_{d}^{*}$; (b) $\bar{q}_{i}^{*}>q_{i}^{*}$ and $\bar{q}_{d}^{*} \geq q_{d}^{*}$.

2) when $\Delta c_{i}<0, \Delta c_{d}<0$, the following scenarios will not happen to the optimal order quantities in either the retail channel or the direct channel:

(c) $\bar{q}_{i}^{*} \leq q_{i}^{*}$ and $\bar{q}_{d}^{*}<q_{d}^{*}$; (d) $\bar{q}_{i}^{*}<q_{i}^{*}$ and $\bar{q}_{d}^{*} \leq q_{d}^{*}$.

Proof Proof by contradiction is used. First, conclusion 1 is proved as follows: 
When $\Delta c_{i}>0, \Delta c_{d}>0$, assume there is (a) $\bar{q}_{i}^{*} \geq q_{i}^{*}$ and $\bar{q}_{d}^{*}>q_{d}^{*}$ for the optimal order quantities of the retail channel and the direct channel. In normal situations, $q_{i}^{*}$ and $q_{d}^{*}$ represent the optimal order quantities of the two channels. Therefore, there is $\pi\left(q_{i}, q_{d}\right) \leq \pi\left(q_{i}^{*}, q_{d}^{*}\right)$.

Based on the assumptions, when the optimal order quantities are $\bar{q}_{i}^{*}$ and $\bar{q}_{d}^{*}$ in the two channels after cost disruption, the optimal profit function of an integrated supply chain is

$$
\bar{\pi}\left(\bar{q}_{i}, \bar{q}_{d}\right)=\pi\left(\bar{q}_{i}^{*}, \bar{q}_{d}^{*}\right)-\Delta c_{i} \bar{q}_{i}^{*}-\Delta c_{d} \bar{q}_{d}^{*}-\left[\lambda_{i+}\left(\bar{q}_{i}^{*}-q_{i}^{*}\right)+\lambda_{d+}\left(\bar{q}_{d}^{*}-q_{d}^{*}\right)\right] .
$$

Accordingly, there is

$$
\begin{aligned}
\bar{\pi}\left(\bar{q}_{i}, \bar{q}_{d}\right) & =\pi\left(\bar{q}_{i}^{*}, \bar{q}_{d}^{*}\right)-\Delta c_{i} \bar{q}_{i}^{*}-\Delta c_{d} \bar{q}_{d}^{*}-\left[\lambda_{i+}\left(\bar{q}_{i}^{*}-q_{i}^{*}\right)+\lambda_{d+}\left(\bar{q}_{d}^{*}-q_{d}^{*}\right)\right] \\
& \leq \pi\left(q_{i}^{*}, q_{d}^{*}\right)-\Delta c_{i} \bar{q}_{i}^{*}-\Delta c_{d} \bar{q}_{d}^{*}-\left[\lambda_{i+}\left(\bar{q}_{i}^{*}-q_{i}^{*}\right)+\lambda_{d+}\left(\bar{q}_{d}^{*}-q_{d}^{*}\right)\right] \\
& <\pi\left(q_{i}^{*}, q_{d}^{*}\right)-\Delta c_{i} q_{i}^{*}-\Delta c_{d} q_{d}^{*} \\
& =\bar{\pi}\left(q_{i}^{*}, q_{d}^{*}\right) .
\end{aligned}
$$

This contradicts with the notation that $\bar{q}_{i}^{*}$ and $\bar{q}_{d}^{*}$ are the optimal order quantities in the two channels. Consequently, the assumption is wrong and the proposition is right, i.e., the scenario that $\bar{q}_{i}^{*} \geq q_{i}^{*}$ and $\bar{q}_{d}^{*}>q_{d}^{*}$ will not happen to the optimal order quantities in either the retail channel or the direct channel

For the same reason, other propositions in conclusion 2 can also be proved.

Based on conclusion 2, there is

Proposition 1 When $\Delta c_{i}>0, \Delta c_{d}>0$, the optimization decision of the profit function of an integrated supply chain system can be classified into the following situations:

(I)

$$
\begin{aligned}
& \max _{\left(\bar{q}_{i}, \bar{q}_{d}\right)} \bar{\pi}\left(\bar{q}_{i}, \bar{q}_{d}\right)=\left(p_{i}-c_{i}-\Delta c_{i}\right) \bar{q}_{i}+\left(p_{d}-c_{d}-\Delta c_{d}\right) \bar{q}_{d}-\lambda_{i-}\left(q_{i}^{*}-\bar{q}_{i}\right)-\lambda_{d-}\left(q_{d}^{*}-\bar{q}_{d}\right) \\
& \text { s.t. } q_{i}^{*} \geq \bar{q}_{i}, \quad q_{d}^{*} \geq \bar{q}_{d} .
\end{aligned}
$$

$$
\begin{aligned}
& \max _{\left(\bar{q}_{i}, \bar{q}_{d}\right)} \bar{\pi}\left(\bar{q}_{i}, \bar{q}_{d}\right)=\left(p_{i}-c_{i}-\Delta c_{i}\right) \bar{q}_{i}+\left(p_{d}-c_{d}-\Delta c_{d}\right) \bar{q}_{d}-\lambda_{i+}\left(\bar{q}_{i}-q_{i}^{*}\right)-\lambda_{d-}\left(q_{d}^{*}-\bar{q}_{d}\right) \\
& \text { s.t. } q_{i}^{*} \leq \bar{q}_{i}, \quad q_{d}^{*} \geq \bar{q}_{d} .
\end{aligned}
$$

$$
\begin{aligned}
& \max _{\left(\bar{q}_{i}, \bar{q}_{d}\right)} \bar{\pi}\left(\bar{q}_{i}, \bar{q}_{d}\right)=\left(p_{i}-c_{i}-\Delta c_{i}\right) \bar{q}_{i}+\left(p_{d}-c_{d}-\Delta c_{d}\right) \bar{q}_{d}-\lambda_{i-}\left(q_{i}^{*}-\bar{q}_{i}\right)-\lambda_{d+}\left(\bar{q}_{d}-q_{d}^{*}\right) \\
& \text { s.t. } q_{i}^{*} \geq \bar{q}_{i}, \quad q_{d}^{*} \leq \bar{q}_{d} .
\end{aligned}
$$

Proposition 2 When $\Delta c_{i}<0, \Delta c_{d}<0$, the optimization decision of the profit function of an integrated supply chain system can be classified into the following situations:

(IV)

$$
\begin{aligned}
& \max _{\left(\bar{q}_{i}, \bar{q}_{d}\right)} \bar{\pi}\left(\bar{q}_{i}, \bar{q}_{d}\right)=\left(p_{i}-c_{i}-\Delta c_{i}\right) \bar{q}_{i}+\left(p_{d}-c_{d}-\Delta c_{d}\right) \bar{q}_{d}-\lambda_{i+}\left(\bar{q}_{i}-q_{i}^{*}\right)-\lambda_{d+}\left(\bar{q}_{d}-q_{d}^{*}\right) \\
& \text { s.t. } q_{i}^{*} \leq \bar{q}_{i}, \quad q_{d}^{*} \leq \bar{q}_{d} .
\end{aligned}
$$


$(\mathrm{V})$

$$
\begin{aligned}
& \max _{\left(\bar{q}_{i}, \bar{q}_{d}\right)} \bar{\pi}\left(\bar{q}_{i}, \bar{q}_{d}\right)=\left(p_{i}-c_{i}-\Delta c_{i}\right) \bar{q}_{i}+\left(p_{d}-c_{d}-\Delta c_{d}\right) \bar{q}_{d}-\lambda_{i+}\left(\bar{q}_{i}-q_{i}^{*}\right)-\lambda_{d-}\left(q_{d}^{*}-\bar{q}_{d}\right) \\
& \text { s.t. } q_{i}^{*} \leq \bar{q}_{i}, \quad q_{d}^{*} \geq \bar{q}_{d} \text {. }
\end{aligned}
$$

$$
\begin{aligned}
& \max _{\left(\bar{q}_{i}, \bar{q}_{d}\right)} \bar{\pi}\left(\bar{q}_{i}, \bar{q}_{d}\right)=\left(p_{i}-c_{i}-\Delta c_{i}\right) \bar{q}_{i}+\left(p_{d}-c_{d}-\Delta c_{d}\right) \bar{q}_{d}-\lambda_{i-}\left(q_{i}^{*}-\bar{q}_{i}\right)-\lambda_{d+}\left(\bar{q}_{d}-q_{d}^{*}\right) \\
& \text { s.t. } q_{i}^{*} \geq \bar{q}_{i}, \quad q_{d}^{*} \leq \bar{q}_{d} \text {. }
\end{aligned}
$$

Next, the optimization problem of supply chain profit function under three situations in Proposition 1 are to be solved.

Let the optimal solutions of (I) in Proposition 1 be $\left(\bar{q}_{i}^{*}, \bar{q}_{d}^{*}\right)$, and Lagrange multipliers $\xi_{1}$ and $\xi_{2}$ are introduced, then K-T conditions can be obtained as follows:

$$
\left\{\begin{array}{l}
\partial \bar{\pi} / \partial \bar{q}_{i}-\xi_{1}=0, \\
\partial \bar{\pi} / \partial \bar{q}_{d}-\xi_{2}=0, \quad \text { and } \quad q_{i}^{*} \geq q_{i} \geq 0, \quad q_{d}^{*} \geq q_{d} \geq 0, \quad \xi_{1} \geq 0, \quad \xi_{2} \geq 0 . \\
\xi_{1}\left(q_{i}^{*}-\bar{q}_{i}\right)=0, \\
\xi_{2}\left(q_{d}^{*}-\bar{q}_{d}\right)=0,
\end{array}\right.
$$

Solution of Formula (19) yields:

When $0 \leq \Delta c_{i}<\lambda_{i-}, 0 \leq \Delta c_{d}<\lambda_{d-}$,

$$
\bar{q}_{i}^{*}=q_{i}^{*}, \quad \bar{q}_{d}^{*}=q_{d}^{*}
$$

When $\Delta c_{i} \geq \lambda_{i_{-}}, 0 \leq \Delta c_{d}<\beta \Delta c_{i}+\lambda_{d-}-\beta \lambda_{i-}$,

$$
\bar{q}_{i}^{*}=q_{i}^{*}-\frac{\left(1-\beta^{2}\right)\left(\Delta c_{i}-\lambda_{i-}\right)}{2}, \quad \bar{q}_{d}^{*}=q_{d}^{*} ;
$$

When $\Delta c_{d} \geq \lambda_{d-}, 0 \leq \Delta c_{i}<\beta \Delta c_{d}+\lambda_{i_{-}}-\beta \lambda_{d-}$,

$$
\bar{q}_{i}^{*}=q_{i}^{*}, \quad \bar{q}_{d}^{*}=q_{d}^{*}-\frac{\left(1-\beta^{2}\right)\left(\Delta c_{d}-\lambda_{d-}\right)}{2} ;
$$

When $\Delta c_{i} \geq \lambda_{i-}, \Delta c_{i} \geq \beta \Delta c_{d}+\lambda_{i-}-\beta \lambda_{d-}$ and $\Delta c_{d} \geq \lambda_{d-}, \Delta c_{d} \geq \beta \Delta c_{i}+\lambda_{d_{-}}-\beta \lambda_{i-}$,

$$
\bar{q}_{i}^{*}=q_{i}^{*}+\frac{\beta \Delta c_{d}-\Delta c_{i}+\lambda_{i-}-\beta \lambda_{d-}}{2}, \quad \bar{q}_{d}^{*}=q_{d}^{*}+\frac{\beta \Delta c_{i}-\Delta c_{d}+\lambda_{d-}-\beta \lambda_{i-}}{2} .
$$

In the same way, the optimal solutions $\left(\bar{q}_{i}^{*}, \bar{q}_{d}^{*}\right)$ of (II) in Proposition 1 are as follows: When $0 \leq \Delta c_{i}, 0 \leq \Delta c_{d}<\lambda_{d-}$,

$$
\bar{q}_{i}^{*}=q_{i}^{*}, \quad \bar{q}_{d}^{*}=q_{d}^{*}
$$

When $0 \leq \Delta c_{i} \leq \beta \Delta c_{d}-\lambda_{i+}-\beta \lambda_{d-}, \Delta c_{d} \geq \lambda_{d-}$,

$$
\bar{q}_{i}^{*}=q_{i}^{*}+\frac{\beta \Delta c_{d}-\Delta c_{i}-\lambda_{i+}-\beta \lambda_{d-}}{2}, \quad \bar{q}_{d}^{*}=q_{d}^{*}+\frac{\beta \Delta c_{i}-\Delta c_{d}+\lambda_{d-}+\beta \lambda_{i+}}{2} ;
$$


When $\Delta c_{i}>\max \left\{\beta \Delta c_{d}-\lambda_{i+}-\beta \lambda_{d-}, 0\right\}, \Delta c_{d} \geq \lambda_{d-}$,

$$
\bar{q}_{i}^{*}=q_{i}^{*}, \quad \bar{q}_{d}^{*}=q_{d}^{*}-\frac{\left(1-\beta^{2}\right)\left(\Delta c_{d}-\lambda_{d-}\right)}{2} .
$$

The optimal solutions $\left(\bar{q}_{i}^{*}, \bar{q}_{d}^{*}\right)$ of (III) in Proposition 1 are as follows:

When $0 \leq \Delta c_{i}<\lambda_{i-}, 0 \leq \Delta c_{d}$,

$$
\bar{q}_{i}^{*}=q_{i}^{*}, \quad \bar{q}_{d}^{*}=q_{d}^{*}
$$

When $\Delta c_{i} \geq \lambda_{i-}, 0 \leq \Delta c_{d} \leq \beta \Delta c_{i}-\lambda_{d+}-\beta \lambda_{i-}$,

$$
\bar{q}_{i}^{*}=q_{i}^{*}+\frac{\beta \Delta c_{d}-\Delta c_{i}+\lambda_{i-}+\beta \lambda_{d+}}{2}, \quad \bar{q}_{d}^{*}=q_{d}^{*}+\frac{\beta \Delta c_{i}-\Delta c_{d}-\lambda_{d+}-\beta \lambda_{i-}}{2} ;
$$

When $\Delta c_{i} \geq \lambda_{i-}, \Delta c_{d} \geq \max \left\{\beta \Delta c_{i}-\lambda_{d+}-\beta \lambda_{i_{-}}, 0\right\}$,

$$
\bar{q}_{i}^{*}=q_{i}^{*}-\frac{\left(1-\beta^{2}\right)\left(\Delta c_{i}-\lambda_{i-}\right)}{2}, \quad \bar{q}_{d}^{*}=q_{d}^{*} .
$$

Likewise, the optimal solutions $\left(\bar{q}_{i}^{*}, \bar{q}_{d}^{*}\right)$ of (IV) in Proposition 2 are as follows:

When $0>\Delta c_{i}>-\lambda_{i+}, 0>\Delta c_{d}>-\lambda_{d+}$,

$$
\bar{q}_{i}^{*}=q_{i}^{*}, \quad \bar{q}_{d}^{*}=q_{d}^{*} ;
$$

When $\Delta c_{i} \leq-\lambda_{i+}, 0>\Delta c_{d}>\beta \Delta c_{i}-\lambda_{d+}+\beta \lambda_{i+}$,

$$
\bar{q}_{i}^{*}=q_{i}^{*}-\frac{\left(1-\beta^{2}\right)\left(\Delta c_{i}+\lambda_{i+}\right)}{2}, \quad \bar{q}_{d}^{*}=q_{d}^{*} ;
$$

When $\Delta c_{d} \leq-\lambda_{d+}, 0>\Delta c_{i}>\beta \Delta c_{d}-\lambda_{i+}+\beta \lambda_{d+}$,

$$
\bar{q}_{i}^{*}=q_{i}^{*}, \quad \bar{q}_{d}^{*}=q_{d}^{*}-\frac{\left(1-\beta^{2}\right)\left(\Delta c_{d}+\lambda_{d+}\right)}{2} ;
$$

When $\Delta c_{i} \leq-\lambda_{i+}, \Delta c_{i} \leq \beta \Delta c_{d}-\lambda_{i+}+\beta \lambda_{d+}$ and $\Delta c_{d} \leq-\lambda_{d+}, \Delta c_{d} \leq \beta \Delta c_{i}-\lambda_{d+}+\beta \lambda_{i+}$,

$$
\bar{q}_{i}^{*}=q_{i}^{*}+\frac{\beta \Delta c_{d}-\Delta c_{i}-\lambda_{i+}+\beta \lambda_{d+}}{2}, \quad \bar{q}_{d}^{*}=q_{d}^{*}+\frac{\beta \Delta c_{i}-\Delta c_{d}-\lambda_{d+}+\beta \lambda_{i+}}{2} .
$$

The optimal solutions $\left(\bar{q}_{i}^{*}, \bar{q}_{d}^{*}\right)$ of $(\mathrm{V})$ in Proposition 2 are as follows:

When $0>\Delta c_{i}>-\lambda_{i+}, 0>\Delta c_{d}$,

$$
\bar{q}_{i}^{*}=q_{i}^{*}, \quad \bar{q}_{d}^{*}=q_{d}^{*}
$$

When $\Delta c_{i} \leq-\lambda_{i+}, 0>\Delta c_{d} \geq \beta \Delta c_{i}+\lambda_{d-}+\beta \lambda_{i+}$,

$$
\bar{q}_{i}^{*}=q_{i}^{*}+\frac{\beta \Delta c_{d}-\Delta c_{i}-\lambda_{i+}-\beta \lambda_{d-}}{2}, \quad \bar{q}_{d}^{*}=q_{d}^{*}+\frac{\beta \Delta c_{i}-\Delta c_{d}+\lambda_{d-}+\beta \lambda_{i+}}{2} ;
$$

When $\Delta c_{i} \leq-\lambda_{i+}, \Delta c_{d}<\min \left\{\beta \Delta c_{i}+\lambda_{d-}+\beta \lambda_{i+}, 0\right\}$,

$$
\bar{q}_{i}^{*}=q_{i}^{*}-\frac{\left(1-\beta^{2}\right)\left(\Delta c_{i}+\lambda_{i+}\right)}{2}, \quad \bar{q}_{d}^{*}=q_{d}^{*} .
$$

The optimal solutions $\left(\bar{q}_{i}^{*}, \bar{q}_{d}^{*}\right)$ of (VI) in Proposition 2 are as follows: 
When $0>\Delta c_{i}, 0>\Delta c_{d}>-\lambda_{d+}$,

$$
\bar{q}_{i}^{*}=q_{i}^{*}, \quad \bar{q}_{d}^{*}=q_{d}^{*}
$$

When $0>\Delta c_{i} \geq \beta \Delta c_{d}+\lambda_{i-}+\beta \lambda_{d+}, \Delta c_{d} \leq-\lambda_{d+}$,

$$
\bar{q}_{i}^{*}=q_{i}^{*}+\frac{\beta \Delta c_{d}-\Delta c_{i}+\lambda_{i-}+\beta \lambda_{d+}}{2}, \quad \bar{q}_{d}^{*}=q_{d}^{*}+\frac{\beta \Delta c_{i}-\Delta c_{d}-\lambda_{d+}-\beta \lambda_{i-}}{2} ;
$$

When $\Delta c_{i} \leq \min \left\{\beta \Delta c_{d}+\lambda_{i-}+\beta \lambda_{d+}, 0\right\}, \Delta c_{d} \leq-\lambda_{d+}$,

$$
\bar{q}_{i}^{*}=q_{i}^{*}, \quad \bar{q}_{d}^{*}=q_{d}^{*}-\frac{\left(1-\beta^{2}\right)\left(\Delta c_{d}+\lambda_{d+}\right)}{2} .
$$

By synthesizing the optimal solutions to the profit function of an integrated supply chain system for the six situations in Proposition 1 and Proposition 2, supply chain strategies to deal with cost disruptions in both channels can be obtained, i.e., Conclusion 3.

Conclusion 3 After product cost is disrupted in an integrated supply chain, the manufacturer's optimal output $\bar{q}^{*}$, the retailer's optimal order quantity $\bar{q}_{i}^{*}$, the optimal order quantity $\bar{q}_{d}^{*}$ in the online direct channel, as well as the optimal sales price $\bar{p}_{i}^{*}$ in the traditional retail channel, and the optimal sales price $\bar{p}_{d}^{*}$ in the direct channel, are respectively as follows:

(I) When product costs in both channels increase, i.e., $\Delta c_{i}>0, \Delta c_{d}>0$, there is

(A) When $0 \leq \Delta c_{i}<\lambda_{i-}, 0 \leq \Delta c_{d}<\lambda_{d-}$,

$$
\begin{array}{ll}
\bar{q}^{*}=q^{*} ; & \\
\bar{q}_{i}^{*}=q_{i}^{*}, & \bar{q}_{d}^{*}=q_{d}^{*} ; \\
\bar{p}_{i}^{*}=p_{i}^{*}, & \bar{p}_{d}^{*}=p_{d}^{*} .
\end{array}
$$

Here, the optimal profit $\bar{\pi}^{*}$ of an integrated supply chain system is

$$
\bar{\pi}^{*}=\pi^{*}-\Delta c_{i} q_{i}^{*}-\Delta c_{d} q_{d}^{*}
$$

(B) When $\Delta c_{i} \geq \lambda_{i-}$ and $0 \leq \Delta c_{d}<\beta \Delta c_{i}-\lambda_{d+}-\beta \lambda_{i-}$,

$$
\begin{aligned}
& \bar{q}^{*}=q^{*}-\frac{(1-\beta)\left(\Delta c_{d}+\Delta c_{i}-\lambda_{i-}+\lambda_{d+}\right)}{2}, \\
& \bar{q}_{i}^{*}=q_{i}^{*}+\frac{\beta \Delta c_{d}-\Delta c_{i}+\lambda_{i-}+\beta \lambda_{d+}}{2}, \quad \bar{q}_{d}^{*}=q_{d}^{*}+\frac{\beta \Delta c_{i}-\Delta c_{d}-\lambda_{d+}-\beta \lambda_{i-}}{2}, \\
& \bar{p}_{i}^{*}=p_{i}^{*}+\frac{\Delta c_{i}-\lambda_{i-}}{2}, \quad \bar{p}_{d}^{*}=p_{d}^{*}+\frac{\Delta c_{d}+\lambda_{d+}}{2} .
\end{aligned}
$$

Here, the optimal profit $\bar{\pi}^{*}$ of an integrated supply chain system is

$$
\begin{aligned}
\bar{\pi}^{*}= & \pi^{*}-\frac{\left(\Delta c_{i}+\lambda_{i-}\right) q_{i}^{*}}{2}-\frac{\left(\Delta c_{d}-\lambda_{d+}\right) q_{d}^{*}}{2} \\
& +\frac{\left(\beta \Delta c_{d}-\Delta c_{i}+\lambda_{i-}+\beta \lambda_{d+}\right)}{2}\left(p_{i}^{*}-\frac{\Delta c_{i}-\lambda_{i-}+2 c_{i}}{2}\right) \\
& +\frac{\left(\beta \Delta c_{i}-\Delta c_{d}-\lambda_{d+}-\beta \lambda_{i-}\right)\left(p_{d}^{*}-\frac{\Delta c_{d}+\lambda_{d+}+2 c_{d}}{2}\right)}{2}
\end{aligned}
$$


(C) When $\Delta c_{i} \geq \lambda_{i-}$ and $\max \left\{0, \beta \Delta c_{i}-\lambda_{d+}-\beta \lambda_{i-}\right\} \leq \Delta c_{d}<\beta \Delta c_{i}+\lambda_{d_{-}}-\beta \lambda_{i_{-}}$,

$$
\begin{aligned}
& \bar{q}^{*}=q^{*}-\frac{\left(1-\beta^{2}\right)\left(\Delta c_{i}-\lambda_{i-}\right)}{2}, \\
& \bar{q}_{i}^{*}=q_{i}^{*}-\frac{\left(1-\beta^{2}\right)\left(\Delta c_{i}-\lambda_{i-}\right)}{2}, \quad \bar{q}_{d}^{*}=q_{d}^{*}, \\
& \bar{p}_{i}^{*}=p_{i}^{*}+\frac{\Delta c_{i}-\lambda_{i-}}{2}, \quad \bar{p}_{d}^{*}=p_{d}^{*}+\frac{\beta\left(\Delta c_{i}-\lambda_{i-}\right)}{2} .
\end{aligned}
$$

Here, the optimal profit $\bar{\pi}^{*}$ of an integrated supply chain system is

$$
\begin{aligned}
\bar{\pi}^{*}= & \pi^{*}-\frac{\left(\Delta c_{i}+\lambda_{i-}\right) q_{i}^{*}}{2}+\frac{\left(\beta \Delta c_{i}-\beta \lambda_{i-}-2 \Delta c_{d}\right) q_{d}^{*}}{2} \\
& -\frac{\left(1-\beta^{2}\right)\left(\Delta c_{i}-\lambda_{i-}\right)}{2}\left(p_{i}^{*}-\frac{\Delta c_{i}-\lambda_{i-}+2 c_{i}}{2}\right) .
\end{aligned}
$$

(D) When $0 \leq \Delta c_{i} \leq \beta \Delta c_{d}-\lambda_{i+}-\beta \lambda_{d-}, \Delta c_{d} \geq \lambda_{d_{-}}$,

$$
\begin{aligned}
& \bar{q}^{*}=q^{*}-\frac{(1-\beta)\left(\Delta c_{d}+\Delta c_{i}+\lambda_{i+}-\lambda_{d-}\right)}{2}, \\
& \bar{q}_{i}^{*}=q_{i}^{*}+\frac{\beta \Delta c_{d}-\Delta c_{i}-\lambda_{i+}-\beta \lambda_{d-}}{2}, \quad \bar{q}_{d}^{*}=q_{d}^{*}+\frac{\beta \Delta c_{i}-\Delta c_{d}+\lambda_{d-}+\beta \lambda_{i+}}{2}, \\
& \bar{p}_{i}^{*}=p_{i}^{*}+\frac{\Delta c_{i}+\lambda_{i+}}{2}, \quad \bar{p}_{d}^{*}=p_{d}^{*}+\frac{\Delta c_{d}-\lambda_{d-}}{2} .
\end{aligned}
$$

Here, the optimal profit $\bar{\pi}^{*}$ of an integrated supply chain system is

$$
\begin{aligned}
\bar{\pi}^{*}= & \pi^{*}-\frac{\left(\Delta c_{i}-\lambda_{i+}\right) q_{i}^{*}}{2}-\frac{\left(\Delta c_{d}+\lambda_{d-}\right) q_{d}^{*}}{2} \\
& +\frac{\left(\beta \Delta c_{d}-\Delta c_{i}-\lambda_{i+}-\beta \lambda_{d-}\right)}{2}\left(p_{i}^{*}-\frac{\Delta c_{i}+\lambda_{i+}+2 c_{i}}{2}\right) \\
& +\frac{\left(\beta \Delta c_{i}-\Delta c_{d}+\lambda_{d-}+\beta \lambda_{i+}\right)\left(p_{d}^{*}-\frac{\Delta c_{d}-\lambda_{d-}+2 c_{d}}{2}\right)}{2} .
\end{aligned}
$$

(E) When $0 \leq \Delta c_{i}, \Delta c_{d} \geq \lambda_{d_{-}}, \beta \Delta c_{d}-\lambda_{i+}-\beta \lambda_{d_{-}}<\Delta c_{i}<\beta \Delta c_{d}+\lambda_{i_{-}}-\beta \lambda_{d-}$,

$$
\begin{aligned}
& \bar{q}^{*}=q^{*}-\frac{\left(1-\beta^{2}\right)\left(\Delta c_{d}-\lambda_{d-}\right)}{2}, \\
& \bar{q}_{i}^{*}=q_{i}^{*}, \quad \bar{q}_{d}^{*}=q_{d}^{*}-\frac{\left(1-\beta^{2}\right)\left(\Delta c_{d}-\lambda_{d-}\right)}{2}, \\
& \bar{p}_{i}^{*}=p_{i}^{*}+\frac{\beta\left(\Delta c_{d}-\lambda_{d-}\right)}{2}, \quad \bar{p}_{d}^{*}=p_{d}^{*}+\frac{\Delta c_{d}-\lambda_{d-}}{2} .
\end{aligned}
$$

Here, the optimal profit $\bar{\pi}^{*}$ of an integrated supply chain system is

$$
\begin{aligned}
\bar{\pi}^{*}= & \pi^{*}+\frac{\left(\beta \Delta c_{d}-\beta \lambda_{d-}-2 \Delta c_{i}\right) q_{i}^{*}}{2}-\frac{\left(\Delta c_{d}+\lambda_{d-}\right) q_{d}^{*}}{2} \\
& -\frac{\left(1-\beta^{2}\right)\left(\Delta c_{d}-\lambda_{d-}\right)}{2}\left(p_{d}^{*}-\frac{\Delta c_{d}-\lambda_{d-}+2 c_{d}}{2}\right) .
\end{aligned}
$$

(F) When $\lambda_{i_{-}} \leq \Delta c_{i}, \lambda_{d-} \leq \Delta c_{d}$ and $\Delta c_{i} \geq \beta \Delta c_{d}+\lambda_{i-}-\beta \lambda_{d-}, \Delta c_{d} \geq \beta \Delta c_{i}-\beta \lambda_{i-}+\lambda_{d-}$,

$$
\bar{q}^{*}=q^{*}-\frac{(1-\beta)\left(\Delta c_{d}+\Delta c_{i}-\lambda_{i-}-\lambda_{d-}\right)}{2},
$$




$$
\begin{aligned}
& \bar{q}_{i}^{*}=q_{i}^{*}+\frac{\beta \Delta c_{d}-\Delta c_{i}+\lambda_{i-}-\beta \lambda_{d-}}{2}, \quad \bar{q}_{d}^{*}=q_{d}^{*}+\frac{\beta \Delta c_{i}-\Delta c_{d}+\lambda_{d-}-\beta \lambda_{i-}}{2}, \\
& \bar{p}_{i}^{*}=p_{i}^{*}+\frac{\Delta c_{i}-\lambda_{i-}}{2}, \quad \bar{p}_{d}^{*}=p_{d}^{*}+\frac{\Delta c_{d}-\lambda_{d-}}{2} .
\end{aligned}
$$

Here, the optimal profit $\bar{\pi}^{*}$ of an integrated supply chain system is

$$
\begin{aligned}
\bar{\pi}^{*}= & \pi^{*}-\frac{\left(\Delta c_{i}+\lambda_{i-}\right) q_{i}^{*}}{2}-\frac{\left(\Delta c_{d}+\lambda_{d-}\right) q_{d}^{*}}{2} \\
& +\frac{\left(\beta \Delta c_{d}-\Delta c_{i}+\lambda_{i-}-\beta \lambda_{d-}\right)}{2}\left(p_{i}^{*}-\frac{\Delta c_{i}-\lambda_{i-}+2 c_{i}}{2}\right) \\
& +\frac{\left(\beta \Delta c_{i}-\Delta c_{d}+\lambda_{d-}-\beta \lambda_{i-}\right)}{2}\left(p_{d}^{*}-\frac{\Delta c_{d}-\lambda_{d-}+2 c_{d}}{2}\right) .
\end{aligned}
$$

(II) When product costs in both channels decrease, i.e., $\Delta c_{i}<0, \Delta c_{d}<0$, there is

(H) When $0>\Delta c_{i}>-\lambda_{i+}, 0>\Delta c_{d}>-\lambda_{d+}$,

$$
\begin{array}{ll}
\bar{q}^{*}=q^{*}, & \\
\bar{q}_{i}^{*}=q_{i}^{*}, & \bar{q}_{d}^{*}=q_{d}^{*}, \\
\bar{p}_{i}^{*}=p_{i}^{*}, & \bar{p}_{d}^{*}=p_{d}^{*} .
\end{array}
$$

Here, the optimal profit $\bar{\pi}^{*}$ of an integrated supply chain system is

$$
\bar{\pi}^{*}=\pi^{*}-\Delta c_{i} q_{i}^{*}-\Delta c_{d} q_{d}^{*} .
$$

(I) When $\Delta c_{i} \leq-\lambda_{i+}, \beta \Delta c_{i}+\beta \lambda_{i+}+\lambda_{d-} \leq \Delta c_{d}<0$,

$$
\begin{aligned}
& \bar{q}^{*}=q^{*}-\frac{(1-\beta)\left(\Delta c_{d}+\Delta c_{i}+\lambda_{i+}-\lambda_{d-}\right)}{2}, \\
& \bar{q}_{i}^{*}=q_{i}^{*}+\frac{\beta \Delta c_{d}-\Delta c_{i}-\lambda_{i+}-\beta \lambda_{d-}}{2}, \quad \bar{q}_{d}^{*}=q_{d}^{*}+\frac{\beta \Delta c_{i}-\Delta c_{d}+\lambda_{d-}+\beta \lambda_{i+}}{2}, \\
& \bar{p}_{i}^{*}=p_{i}^{*}+\frac{\Delta c_{i}+\lambda_{i+}}{2}, \quad \bar{p}_{d}^{*}=p_{d}^{*}+\frac{\Delta c_{d}-\lambda_{d-}}{2} .
\end{aligned}
$$

Here, the optimal profit $\bar{\pi}^{*}$ of an integrated supply chain system is

$$
\begin{aligned}
\bar{\pi}^{*}= & \pi^{*}-\frac{\left(\Delta c_{i}-\lambda_{i+}\right) q_{i}^{*}}{2}-\frac{\left(\Delta c_{d}+\lambda_{d-}\right) q_{d}^{*}}{2} \\
& +\frac{\left(\beta \Delta c_{d}-\Delta c_{i}-\lambda_{i+}-\beta \lambda_{d-}\right)}{2}\left(p_{i}^{*}-\frac{\Delta c_{i}+\lambda_{i+}+2 c_{i}}{2}\right) \\
& +\frac{\left(\beta \Delta c_{i}-\Delta c_{d}+\lambda_{d-}+\beta \lambda_{i+}\right)}{2}\left(p_{d}^{*}-\frac{\Delta c_{d}-\lambda_{d-}+2 c_{d}}{2}\right) .
\end{aligned}
$$

(J) When $\Delta c_{i} \leq-\lambda_{i+}, \Delta c_{d}<0$ and $\beta \Delta c_{i}-\lambda_{d+}+\beta \lambda_{i+}<\Delta c_{d}<\beta \Delta c_{i}+\lambda_{d-}+\beta \lambda_{i+}$,

$$
\begin{aligned}
& \bar{q}^{*}=q^{*}-\frac{\left(1-\beta^{2}\right)\left(\Delta c_{i}+\lambda_{i+}\right)}{2}, \\
& \bar{q}_{i}^{*}=q_{i}^{*}-\frac{\left(1-\beta^{2}\right)\left(\Delta c_{i}+\lambda_{i+}\right)}{2}, \quad \bar{q}_{d}^{*}=q_{d}^{*}, \\
& \bar{p}_{i}^{*}=p_{i}^{*}+\frac{\Delta c_{i}+\lambda_{i+}}{2}, \quad \bar{p}_{d}^{*}=p_{d}^{*}+\frac{\beta\left(\Delta c_{i}+\lambda_{i+}\right)}{2} .
\end{aligned}
$$


Here, the optimal profit $\bar{\pi}^{*}$ of an integrated supply chain system is,

$$
\begin{aligned}
\bar{\pi}^{*}= & \pi^{*}-\frac{\left(\Delta c_{i}-\lambda_{i+}\right) q_{i}^{*}}{2}+\frac{\left(\beta \Delta c_{i}+\beta \lambda_{i+}-2 \Delta c_{d}\right) q_{d}^{*}}{2} \\
& -\frac{\left(1-\beta^{2}\right)\left(\Delta c_{i}+\lambda_{i+}\right)}{2}\left(p_{i}^{*}-\frac{\Delta c_{i}+\lambda_{i+}+2 c_{i}}{2}\right) .
\end{aligned}
$$

(K) When $\Delta c_{d} \leq-\lambda_{d+}, \beta \Delta c_{d}+\lambda_{i-}+\beta \lambda_{d+} \leq \Delta c_{i}<0$,

$$
\begin{aligned}
& \bar{q}^{*}=q^{*}-\frac{(1-\beta)\left(\Delta c_{d}+\Delta c_{i}-\lambda_{i-}+\lambda_{d+}\right)}{2}, \\
& \bar{q}_{i}^{*}=q_{i}^{*}+\frac{\beta \Delta c_{d}-\Delta c_{i}+\lambda_{i-}+\beta \lambda_{d+}}{2}, \quad \bar{q}_{d}^{*}=q_{d}^{*}+\frac{\beta \Delta c_{i}-\Delta c_{d}-\lambda_{d+}-\beta \lambda_{i-}}{2}, \\
& \bar{p}_{i}^{*}=p_{i}^{*}+\frac{\Delta c_{i}-\lambda_{i-}}{2}, \quad \bar{p}_{d}^{*}=p_{d}^{*}+\frac{\Delta c_{d}+\lambda_{d+}}{2}
\end{aligned}
$$

Here, the optimal profit $\bar{\pi}^{*}$ of an integrated supply chain system is

$$
\begin{aligned}
\bar{\pi}^{*}= & \pi^{*}-\frac{\left(\Delta c_{i}+\lambda_{i-}\right) q_{i}^{*}}{2}-\frac{\left(\Delta c_{d}-\lambda_{d+}\right) q_{d}^{*}}{2} \\
& +\frac{\left(\beta \Delta c_{d}-\Delta c_{i}+\lambda_{i-}+\beta \lambda_{d+}\right)}{2}\left(p_{i}^{*}-\frac{\Delta c_{i}-\lambda_{i-}+2 c_{i}}{2}\right) \\
& +\frac{\left(\beta \Delta c_{i}-\Delta c_{d}-\lambda_{d+}-\beta \lambda_{i-}\right)}{2}\left(p_{d}^{*}-\frac{\Delta c_{d}+\lambda_{d+}+2 c_{d}}{2}\right) .
\end{aligned}
$$

(L) When $\Delta c_{i}<0, \Delta c_{d} \leq-\lambda_{d+}, \beta \Delta c_{d}-\lambda_{i+}+\beta \lambda_{d+}<\Delta c_{i}<\beta \Delta c_{d}+\lambda_{i-}+\beta \lambda_{d+}$,

$$
\begin{aligned}
& \bar{q}^{*}=q^{*}-\frac{\left(1-\beta^{2}\right)\left(\Delta c_{d}+\lambda_{d+}\right)}{2}, \\
& \bar{q}_{i}^{*}=q_{i}^{*}, \quad \bar{q}_{d}^{*}=q_{d}^{*}-\frac{\left(1-\beta^{2}\right)\left(\Delta c_{d}+\lambda_{d+}\right)}{2}, \\
& \bar{p}_{i}^{*}=p_{i}^{*}+\frac{\beta\left(\Delta c_{d}+\lambda_{d+}\right)}{2}, \quad \bar{p}_{d}^{*}=p_{d}^{*}+\frac{\Delta c_{d}+\lambda_{d+}}{2} .
\end{aligned}
$$

Here, the optimal profit $\bar{\pi}^{*}$ of an integrated supply chain system is

$$
\begin{aligned}
\bar{\pi}^{*}= & \pi^{*}+\frac{\left(\beta \Delta c_{d}+\beta \lambda_{d+}-2 \Delta c_{i}\right) q_{i}^{*}}{2}-\frac{\left(\Delta c_{d}-\lambda_{d+}\right) q_{d}^{*}}{2} \\
& -\frac{\left(1-\beta^{2}\right)\left(\Delta c_{d}+\lambda_{d+}\right)}{2}\left(p_{d}^{*}-\frac{\Delta c_{d}+\lambda_{d+}+2 c_{d}}{2}\right) .
\end{aligned}
$$

(M) When $\Delta c_{i} \leq-\lambda_{i+}, \Delta c_{d} \leq-\lambda_{d+}$ and $\Delta c_{i} \leq \beta \Delta c_{d}-\lambda_{i+}+\beta \lambda_{d+}, \Delta c_{d} \leq \beta \Delta c_{i}+$ $\beta \lambda_{i+}-\lambda_{d+}$,

$$
\begin{aligned}
& \bar{q}^{*}=q^{*}-\frac{(1-\beta)\left(\Delta c_{d}+\Delta c_{i}+\lambda_{i+}+\lambda_{d+}\right)}{2}, \\
& \bar{q}_{i}^{*}=q_{i}^{*}+\frac{\beta \Delta c_{d}-\Delta c_{i}-\lambda_{i+}+\beta \lambda_{d+}}{2}, \quad \bar{q}_{d}^{*}=q_{d}^{*}+\frac{\beta \Delta c_{i}-\Delta c_{d}-\lambda_{d+}+\beta \lambda_{i+}}{2}, \\
& \bar{p}_{i}^{*}=p_{i}^{*}+\frac{\Delta c_{i}+\lambda_{i+}}{2}, \quad \bar{p}_{d}^{*}=p_{d}^{*}+\frac{\Delta c_{d}+\lambda_{d+}}{2} .
\end{aligned}
$$

Here, the optimal profit $\bar{\pi}^{*}$ of an integrated supply chain system is

$$
\bar{\pi}^{*}=\pi^{*}-\frac{\left(\Delta c_{i}-\lambda_{i+}\right) q_{i}^{*}}{2}-\frac{\left(\Delta c_{d}-\lambda_{d+}\right) q_{d}^{*}}{2}
$$




$$
\begin{aligned}
& +\frac{\left(\beta \Delta c_{d}-\Delta c_{i}-\lambda_{i+}+\beta \lambda_{d+}\right)}{2}\left(p_{i}^{*}-\frac{\Delta c_{i}+\lambda_{i+}+2 c_{i}}{2}\right) \\
& +\frac{\left(\beta \Delta c_{i}-\Delta c_{d}-\lambda_{d+}+\beta \lambda_{i+}\right)}{2}\left(p_{d}^{*}-\frac{\Delta c_{d}+\lambda_{d+}+2 c_{d}}{2}\right) .
\end{aligned}
$$

Thus, it can be seen, product output, sales price and order quantities in each sales channels change when product cost is disrupted in an integrated supply chain. Consequently, profit of an integrated supply chain will change with the adoption of adjustment strategies.

It follows from Conclusion 3 that compared with production strategies in normal situations, when unforeseen events result in increased product cost, strategies that an integrated supply chain should adopt are as follows:

When product costs in both channels increase moderately, i.e., $0 \leq \Delta c_{i}<\lambda_{i-}, 0 \leq \Delta c_{d}<$ $\lambda_{d-}$, the optimal outputs should be maintained for both channels as they are in normal situations. Sales prices are also to be maintained as in normal situations, i.e., this decision has certain robustness, and robust range is positively correlated with the unit disposal cost of surplus products.

When product cost increases more in the retail channel than that in the direct channel, i.e., $\Delta c_{i} \geq \lambda_{i-}$, the optimal order quantity is to be reduced compared to they are in normal situations, and so is the product output. Meanwhile, sales price in retail channel is to be slightly raised by $\left(\Delta c_{i}-\lambda_{i-}\right) / 2$ than that in normal situations. Here, if product cost in direct channel increases less than $\beta \Delta c_{i}-\lambda_{d+}-\beta \lambda_{i-}$, i.e., $0 \leq \Delta c_{d}<\beta \Delta c_{i}-\lambda_{d+}-\beta \lambda_{i-}$, the order quantity of the direct channel is to be increased by $\left(\beta \Delta c_{i}-\lambda_{d+}-\beta \lambda_{i-}-\Delta c_{d}\right) / 2$, so is product output, and the sales price in the direct channel is to be raised by $\left(\Delta c_{d}+\lambda_{d+}\right) / 2$ than that in normal situations. If product cost in the direct channel increases more than $\beta \Delta c_{i}-\lambda_{d+}-\beta \lambda_{i-}$, and satisfies $\beta \Delta c_{i}-\lambda_{d+}-\beta \lambda_{i-} \leq \Delta c_{d}<\beta \Delta c_{i}+\lambda_{d-}-\beta \lambda_{i-}$, the order quantity of the direct channel should be maintained as that in normal situations, and the sales price is to be raised by $\beta\left(\Delta c_{i}-\lambda_{i-}\right) / 2$.

When product cost increases more in the direct channel than that in the retail channel, i.e., $\Delta c_{d} \geq \lambda_{d-}$, the optimal order quantity in the direct channel is to be reduced compared to they are in normal situations, and so is product output. Meanwhile the sales price in the direct channel is to be slightly raised by $\left(\Delta c_{d}-\lambda_{d-}\right) / 2$ than that in normal situations. Here, if the product cost in the retail channel increases less than $\beta \Delta c_{d}-\lambda_{i+}-\beta \lambda_{d-}$, i.e., $0 \leq \Delta c_{i} \leq \beta \Delta c_{d}-\lambda_{i+}-\beta \lambda_{d-}$, order quantity of retail channel is to be increased by $\left(\beta \Delta c_{d}-\right.$ $\left.\Delta c_{i}-\lambda_{i+}-\beta \lambda_{d-}\right) / 2$, and sales price in retail channel is to be raised by $\left(\Delta c_{i}+\lambda_{i+}\right) / 2$ than that in normal situations; if product cost in retail channel increases more than $\beta \Delta c_{d}-\lambda_{i+}-\beta \lambda_{d-}$, and satisfies $\beta \Delta c_{d}-\lambda_{i+}-\beta \lambda_{d-}<\Delta c_{i}<\beta \Delta c_{d}+\lambda_{i-}-\beta \lambda_{d-}$, the order quantity of the retail channel should be maintained as that in normal situations, and the sales price is to be raised by $\beta\left(\Delta c_{d}-\lambda_{d-}\right) / 2$.

When there are relatively large increases in the product costs in both channels, i.e., $\lambda_{i-} \leq$ $\Delta c_{i}, \lambda_{d-} \leq \Delta c_{d}$ and $\Delta c_{i} \geq \beta \Delta c_{d}+\lambda_{i-}-\beta \lambda_{d-}, \Delta c_{d} \geq \beta \Delta c_{i}-\beta \lambda_{i-}+\lambda_{d_{-}}$, the order quantities of both channels are to be reduced, and the sales prices increased. Here, the sales price in the retail channel is to be increased by $\left(\Delta c_{i}-\lambda_{i-}\right) / 2$, and that in the direct channel is to be increased by $\left(\Delta c_{d}-\lambda_{d-}\right) / 2$. Correspondingly, product output is also to be reduced. 
Likewise, an integrated supply chain should adopt the following strategies when unanticipated events result in reduced product cost:

When there are relatively small reduction of product costs in both channels, i.e., $0>\Delta c_{i}>$ $-\lambda_{i+}, 0>\Delta c_{d}>-\lambda_{d+}$, the optimal output in both channels are to be maintained as in normal situations. Sales prices are also to be maintained as in normal situations, i.e., this decision has certain robustness, and robust range is positively correlated with the unit disposal cost of surplus product.

When product cost in the retail channel is reduced more than that in direct channel, i.e., $\Delta c_{i} \leq-\lambda_{i+}$, the optimal order quantity is to be increased compared to it is in normal situations, and the order quantity increases is affected by the product cost change in the direct channel. When product cost reduction in the direct channel is less than $-\left(\beta \Delta c_{i}+\beta \lambda_{i+}+\lambda_{d-}\right)$, i.e., $\beta \Delta c_{i}+\beta \lambda_{i+}+\lambda_{d-} \leq \Delta c_{d}<0$, the order quantity in the retail channel is to be increased by $\left(\beta \Delta c_{d}-\Delta c_{i}-\lambda_{i+}-\beta \lambda_{d-}\right) / 2$. Meanwhile the sales price in the retail channel is to be reduced by $-\left(\Delta c_{i}+\lambda_{i+}\right) / 2$ than that in normal situations, and at this time the optimal order quantity in the direct channel is to be reduced by $-\left(\beta \Delta c_{i}-\Delta c_{d}+\lambda_{d-}+\beta \lambda_{i+}\right) / 2$. Accordingly, the sales price in the direct channel is to be reduced by $\left(\lambda_{d-}-\Delta c_{d}\right) / 2$. When product cost reduction in the direct channel is more than $-\left(\beta \Delta c_{i}+\beta \lambda_{i+}+\lambda_{d-}\right)$, and satisfies $\beta \Delta c_{i}-\lambda_{d+}+\beta \lambda_{i+}<$ $\Delta c_{d}<\beta \Delta c_{i}+\lambda_{d-}+\beta \lambda_{i+}$, the order quantity in the retail channel is to be increased by $-\left(1-\beta^{2}\right)\left(\Delta c_{i}+\lambda_{i+}\right) / 2$. Here, the sales price in the retail channel is still to be reduced by $-\left(\Delta c_{i}+\lambda_{i+}\right) / 2$, but the order quantity in the direct channel is to be maintained. A sales price reduction of $-\beta\left(\Delta c_{i}+\lambda_{i+}\right) / 2$ in the direct channel alone can mitigate the influence of cost disruption.

When product cost in the direct channel is reduced more than that in the direct channel, i.e., $\Delta c_{d} \leq-\lambda_{d+}$, the order quantity in the direct channel is to be increased, and the order quantity increase is affected by the product cost change in the retail channel. When product cost reduction in the direct channel is less than $-\left(\beta \Delta c_{d}+\lambda_{i-}+\beta \lambda_{d+}\right)$, i.e., $\beta \Delta c_{d}+\lambda_{i-}+\beta \lambda_{d+} \leq$ $\Delta c_{i}<0$, the order quantity in the direct channel is to increase by $\left(\beta \Delta c_{i}-\Delta c_{d}-\lambda_{d+}-\beta \lambda_{i-}\right) / 2$. Meanwhile, the sales price in the direct channel is to be reduced by $-\left(\Delta c_{d}+\lambda_{d+}\right) / 2$ than that in normal situations, and at this time the optimal order quantity in the retail channel is to be reduced by $-\left(\beta \Delta c_{d}-\Delta c_{i}+\lambda_{i-}+\beta \lambda_{d+}\right) / 2$. Meanwhile, the sales price in the retail channel is to be reduced by $\left(\lambda_{i-}-\Delta c_{i}\right) / 2$. But when product cost reduction in the retail channel is more than $-\left(\beta \Delta c_{d}+\lambda_{i-}+\beta \lambda_{d+}\right)$, and satisfies $\beta \Delta c_{d}-\lambda_{i+}+\beta \lambda_{d+}<\Delta c_{i}<\beta \Delta c_{d}+\lambda_{i-}+\beta \lambda_{d+}$, the order quantity in the direct channel is to be increased by $-\left(1-\beta^{2}\right)\left(\Delta c_{d}+\lambda_{d+}\right) / 2$. And the sales price in the direct channel is still to be reduced by $-\left(\Delta c_{d}+\lambda_{d+}\right) / 2$. A sales price reduction of $-\beta\left(\Delta c_{i}+\lambda_{i+}\right) / 2$ in the retail channel alone can mitigate the influence of cost disruption.

When product costs in both channels are reduced significantly, i.e., $\Delta c_{i} \leq-\lambda_{i+}, \Delta c_{d} \leq$ $-\lambda_{d+}$ and $\Delta c_{i} \leq \beta \Delta c_{d}-\lambda_{i+}+\beta \lambda_{d+}, \Delta c_{d} \leq \beta \Delta c_{i}+\beta \lambda_{i+}-\lambda_{d+}$, the order quantities of both channels are to be increased, and the sales prices lowered. Here, the sales price in the retail channel is to be reduced by $-\left(\Delta c_{i}+\lambda_{i+}\right) / 2$, and that in the direct channel is to be lowered by $-\left(\Delta c_{d}+\lambda_{d+}\right) / 2$. Consequently, the product output is to be raised. 


\section{Numerical Analysis}

To further analyze the validity of the adjustment strategies adopted by an integrated supply chain to deal with unanticipated events, we take a large-scale enterprise that produces meat products in Shandong, China as a case. This enterprise is also the core enterprise in the supply chain. At present, two sales channels exist in the enterprise, i.e., the traditional retail channel and the online direct sales channel. Through retailers' joining, the enterprise formed an integrated supply chain with the retailers. Fresh pork is used as raw materials in the enterprise. Since the supply of pork is affected by many uncertain factors, sudden pork price fluctuations are frequent in China. This results in sudden product cost fluctuations in the enterprise. Here the models in this paper and the practical operational data of the enterprises are combined, and related operational data are

$$
\alpha_{i}=25, \quad \alpha_{d}=15, \quad c_{i}=21, \quad c_{d}=14, \quad \beta=0.5, \quad \lambda_{i+}=\lambda_{d+}=\lambda_{i-}=\lambda_{d-}=2 .
$$

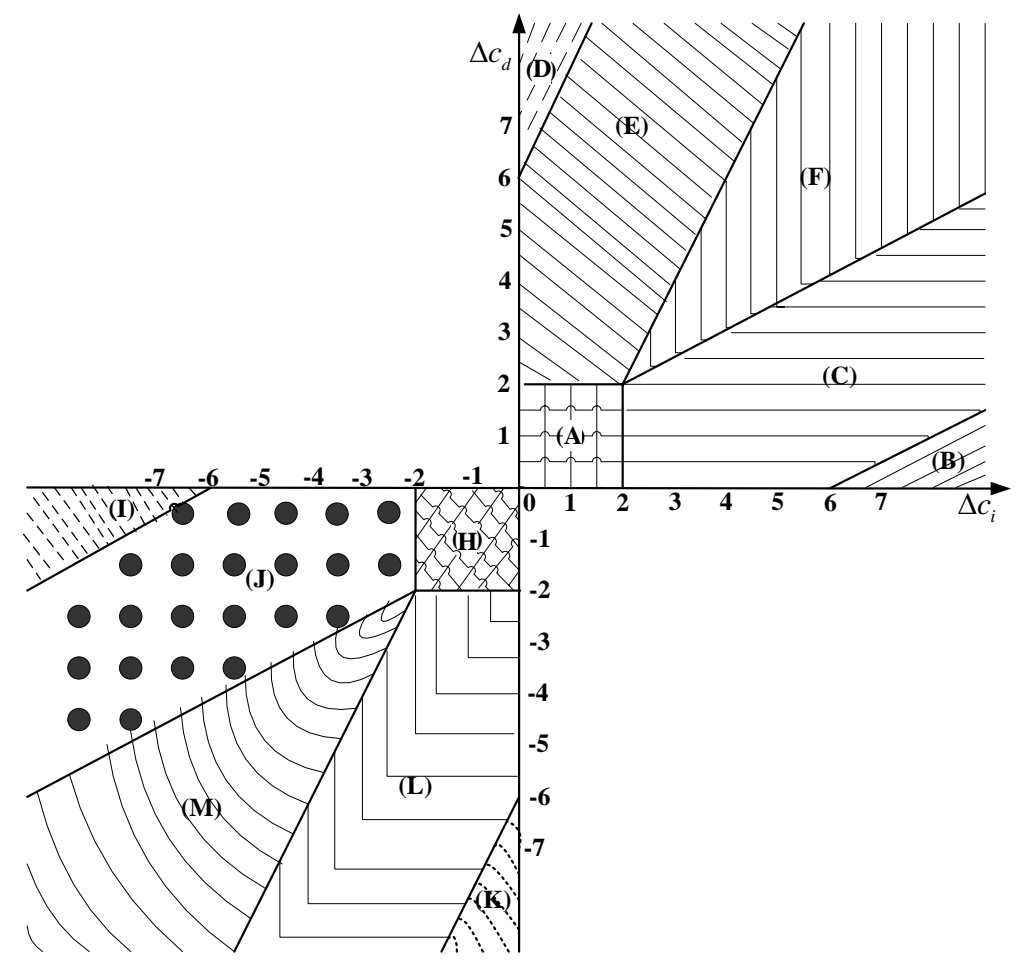

Figure 2 Area map demonstrating the adjustment strategies adopted by an integrated supply chain to cope with cost disruptions

In normal situations, for the integrated supply chain with this company as the core enterprise, the optimal prices in the retail channel and the direct channel are respectively $p_{i}^{*}=32.167$ and $p_{d}^{*}=25.333$; the optimal order quantities in the retail channel and the direct channel are respectively $q_{i}^{*}=5.5$ and $q_{d}^{*}=5.75$; the optimal output of the enterprise is $q^{*}=11.25$, and the optimal system profit of the integrated supply chain is $\pi^{*}=126.583$.

The area map demonstrating the adjustment strategies adopted by the supply chain to cope with cost disruptions is illustrated in Figure 2, based on Conclusion 3. 
When cost disruptions of the supply chain with this enterprise as the core are located in area (A) in Figure 2, the adjustment strategies adopted by the integrated supply chain to cope with cost disruptions are

$$
\begin{array}{ll}
\bar{q}_{i}^{*}=q_{i}^{*}, & \bar{q}_{d}^{*}=q_{d}^{*}, \\
\bar{p}_{i}^{*}=p_{i}^{*}, & \bar{p}_{d}^{*}=p_{d}^{*} .
\end{array}
$$

When cost disruptions are located in area (B) in Figure 2, the adjustment strategies adopted by the system to cope with cost disruptions are

$$
\begin{aligned}
& \bar{q}_{i}^{*}=q_{i}^{*}+0.25 \Delta c_{d}-0.5 \Delta c_{i}+1.5, \quad \bar{q}_{d}^{*}=q_{d}^{*}+0.25 \Delta c_{i}-0.5 \Delta c_{d}-1.5 \\
& \bar{p}_{i}^{*}=p_{i}^{*}+0.5 \Delta c_{i}-1, \quad \bar{p}_{d}^{*}=p_{d}^{*}+0.5 \Delta c_{d}+1 .
\end{aligned}
$$

When cost disruptions are located in area (C) in Figure 2, the adjustment strategies adopted by the system to cope with cost disruptions are

$$
\begin{aligned}
& \bar{q}_{i}^{*}=q_{i}^{*}-0.375 \Delta c_{i}+0.75, \quad \bar{q}_{d}^{*}=q_{d}^{*}, \\
& \bar{p}_{i}^{*}=p_{i}^{*}+0.5 \Delta c_{i}-1, \quad \bar{p}_{d}^{*}=p_{d}^{*}+0.25 \Delta c_{i}-0.5 .
\end{aligned}
$$

When cost disruptions are located in area (D) in Figure 2, the adjustment strategies adopted by the system to cope with cost disruptions are

$$
\begin{aligned}
& \bar{q}_{i}^{*}=q_{i}^{*}+0.25 \Delta c_{d}-0.5 \Delta c_{i}-1.5, \quad \bar{q}_{d}^{*}=q_{d}^{*}+0.25 \Delta c_{i}-0.5 \Delta c_{d}+1.5 \\
& \bar{p}_{i}^{*}=p_{i}^{*}+0.5 \Delta c_{i}+1, \quad \bar{p}_{d}^{*}=p_{d}^{*}+0.5 \Delta c_{d}-1 .
\end{aligned}
$$

When cost disruptions are located in area (E) in Figure 2, the adjustment strategies adopted by the system to cope with cost disruptions are

$$
\begin{aligned}
& \bar{q}_{i}^{*}=q_{i}^{*}, \quad \bar{q}_{d}^{*}=q_{d}^{*}-0.375 \Delta c_{d}+0.75 \\
& \bar{p}_{i}^{*}=p_{i}^{*}+0.25 \Delta c_{d}-0.5, \quad \bar{p}_{d}^{*}=p_{d}^{*}+\Delta c_{d}-1 .
\end{aligned}
$$

When cost disruptions are located in area $(\mathrm{F})$ in Figure 2, the adjustment strategies adopted by the system to cope with cost disruption are

$$
\begin{aligned}
& \bar{q}_{i}^{*}=q_{i}^{*}+0.25 \Delta c_{d}-0.5 \Delta c_{i}+0.5, \quad \bar{q}_{d}^{*}=q_{d}^{*}+0.25 \Delta c_{i}-0.5 \Delta c_{d}+0.5, \\
& \bar{p}_{i}^{*}=p_{i}^{*}+0.5 \Delta c_{i}-1, \quad \bar{p}_{d}^{*}=p_{d}^{*}+0.5 \Delta c_{d}-1 .
\end{aligned}
$$

When cost disruptions are located in area $(\mathrm{H})$ in Figure 2, the adjustment strategies adopted by the system to cope with cost disruptions are

$$
\begin{array}{ll}
\bar{q}_{i}^{*}=q_{i}^{*}, & \bar{q}_{d}^{*}=q_{d}^{*}, \\
\bar{p}_{i}^{*}=p_{i}^{*}, & \bar{p}_{d}^{*}=p_{d}^{*} .
\end{array}
$$

When cost disruptions are located in area $(\mathrm{F})$ in Figure 2, the adjustment strategies adopted by the system to cope with cost disruptions are

$$
\bar{q}_{i}^{*}=q_{i}^{*}+0.25 \Delta c_{d}-0.5 \Delta c_{i}-1.5, \quad \bar{q}_{d}^{*}=q_{d}^{*}+0.25 \Delta c_{i}-0.5 \Delta c_{d}+1.5,
$$




$$
\bar{p}_{i}^{*}=p_{i}^{*}+0.5 \Delta c_{i}+1, \quad \bar{p}_{d}^{*}=p_{d}^{*}+0.5 \Delta c_{d}-1 .
$$

When cost disruptions are located in area $(\mathrm{J})$ in Figure 2, the adjustment strategies adopted by the system to cope with cost disruptions are

$$
\begin{aligned}
& \bar{q}_{i}^{*}=q_{i}^{*}-0.375 \Delta c_{i}-0.75, \quad \bar{q}_{d}^{*}=q_{d}^{*}, \\
& \bar{p}_{i}^{*}=p_{i}^{*}+0.5 \Delta c_{i}+1, \quad \bar{p}_{d}^{*}=p_{d}^{*}+0.25 \Delta c_{d}+0.5 .
\end{aligned}
$$

When cost disruptions are located in area $(\mathrm{K})$ in Figure 2, the adjustment strategies adopted by the system to cope with cost disruptions are

$$
\begin{aligned}
& \bar{q}_{i}^{*}=q_{i}^{*}+0.25 \Delta c_{d}-0.5 \Delta c_{i}+1.5, \quad \bar{q}_{d}^{*}=q_{d}^{*}+0.25 \Delta c_{i}-0.5 \Delta c_{d}-1.5, \\
& \bar{p}_{i}^{*}=p_{i}^{*}+0.5 \Delta c_{i}-1, \quad \bar{p}_{d}^{*}=p_{d}^{*}+0.5 \Delta c_{d}+1 .
\end{aligned}
$$

When cost disruptions are located in area (L) in Figure 2, the adjustment strategies adopted by the system to cope with cost disruptions are

$$
\begin{aligned}
& \bar{q}_{i}^{*}=q_{i}^{*}, \quad \bar{q}_{d}^{*}=q_{d}^{*}-0.375 \Delta c_{d}-0.75, \\
& \bar{p}_{i}^{*}=p_{i}^{*}+0.25 \Delta c_{d}+0.5, \quad \bar{p}_{d}^{*}=p_{d}^{*}+0.5 \Delta c_{d}+1 .
\end{aligned}
$$

When cost disruptions are located in area $(\mathrm{M})$ in Figure 2, the adjustment strategies adopted by the system to cope with cost disruptions are

$$
\begin{aligned}
& \bar{q}_{i}^{*}=q_{i}^{*}+0.25 \Delta c_{d}-0.5 \Delta c_{i}-0.5, \quad \bar{q}_{d}^{*}=q_{d}^{*}+0.25 \Delta c_{i}-0.5 \Delta c_{d}-0.5, \\
& \bar{p}_{i}^{*}=p_{i}^{*}+0.5 \Delta c_{i}+1, \quad \bar{p}_{d}^{*}=p_{d}^{*}+0.5 \Delta c_{d}+1 .
\end{aligned}
$$

When product costs are disrupted, the enterprise shall adopt adjustment strategies as shown in Table 1.

Table 1 has several indications. When product cost increases due to the disruptions of unforeseen events, i.e., $\Delta c_{i}>0, \Delta c_{d}>0$, unanticipated events bring about tremendous adverse effect to the system, and lead to system profit loss. When product cost increases sharply, i.e., $\left(\Delta c_{i}, \Delta c_{d}\right)=(8,8)$, the maximum system profit loss is 81 . But the loss can be mitigated by the adoption of proper strategies. When product cost decreases as a result of the disruptions of unforeseen events, both the production and sales of an integrated supply chain increase, and system profit will increase. Moreover, system profit will increase more, if proper strategies are adopted. For example, When $\left(\Delta c_{i}, \Delta c_{d}\right)=(-10,-3)$, system profit increases to 317.582 , after adoption of proper strategy. This displays that adoption of proper strategies will effectively reduce system loss, whether disruptions cause product cost to increase or decrease. The following are the analysis of strategies in different cost disruption situations in Table 1.

1) When the disruptions of unforeseen events result in moderate product cost increase in both sales channels, i.e., $\left(\Delta c_{i}, \Delta c_{d}\right)=(1,1)$, the optimal output and sales prices should be maintained as in normal situations. But system profit will decrease as product cost increases;

2) When the disruptions of unforeseen events lead to more significant increase of product cost in the retail channel than that in the direct channel, i.e., $\left(\Delta c_{i}, \Delta c_{d}\right)=(10,3)$, the order quantity of the retail channel should be reduced, and the order quantity of the direct channel 
Table 1 The optimal adjustment strategies adopted by an integrated supply chain in different disruption situations

\begin{tabular}{lllllllllll}
\hline $\begin{array}{l}\text { disruption } \\
\text { situations }\end{array}$ & $\Delta c_{i}$ & $\Delta c_{d}$ & $\bar{p}_{i}^{*}$ & $\bar{p}_{d}^{*}$ & $\bar{q}_{i}^{*}$ & $\bar{q}_{d}^{*}$ & $\bar{q}^{*}$ & $\bar{\pi}^{*}$ & $\bar{\pi}^{*}-\pi^{*}$ & $\begin{array}{l}\text { disruption } \\
\text { area }\end{array}$ \\
\hline 1 & 8 & 8 & 35.167 & 28.333 & 4 & 4.25 & 8.25 & 45.583 & -81 & $(\mathrm{~F})$ \\
2 & 3 & 10 & 34.167 & 34.333 & 5.5 & 2.75 & 8.25 & 78.334 & -48.249 & $(\mathrm{E})$ \\
3 & 3 & 13 & 34.667 & 30.833 & 5.75 & 1.5 & 7.25 & 58.085 & -68.498 & $(\mathrm{D})$ \\
4 & 13 & 3 & 37.667 & 27.833 & 1.25 & 5.95 & 7.22 & 102.368 & -24.215 & $(\mathrm{~B})$ \\
5 & 10 & 3 & 36.167 & 27.333 & 2.5 & 5.75 & 8.25 & 98.018 & -28.565 & $(\mathrm{C})$ \\
6 & 1 & 1 & 32.167 & 25.333 & 5.5 & 5.75 & 11.25 & 115.333 & -11.25 & $(\mathrm{~A})$ \\
7 & -1 & -1 & 32.167 & 25.333 & 5.5 & 5.75 & 11.25 & 137.833 & 11.25 & $(\mathrm{H})$ \\
8 & -10 & -3 & 28.167 & 25.083 & 8.5 & 5.75 & 14.25 & 317.582 & 190.999 & $(\mathrm{~J})$ \\
9 & -13 & -3 & 26.667 & 22.833 & 9.75 & 5.5 & 15.25 & 283.355 & 156.772 & $(\mathrm{I})$ \\
10 & -3 & -13 & 29.667 & 19.833 & 5.25 & 10 & 15.25 & 286.936 & 160.353 & $(\mathrm{~K})$ \\
11 & -3 & -10 & 30.167 & 21.333 & 5.5 & 8.75 & 14.75 & 281.352 & 154.769 & $(\mathrm{~L})$ \\
12 & -8 & -8 & 29.167 & 22.333 & 7 & 7.25 & 14.25 & 315.603 & 189.02 & $(\mathrm{M})$ \\
\hline
\end{tabular}

should be maintained. Meanwhile product sales prices in both channels should be raised. Consequently, system profit will fall. The reasons are as follows: large increase of product sales price in the retail channel and moderate increase of product sales price in the direct channel strengthen price advantage of products sold through the direct channel, and cause consumer demand to switch from the retail channel to the direct channel, which results in reduced sales in the retail channel and stable sales in the direct channels.

Likewise, when the disruptions of unforeseen events lead to more significant increase of product cost in the direct channel than that in the retail channel, i.e., $\left(\Delta c_{i}, \Delta c_{d}\right)=(3,10)$, the order quantity of the direct channel should be reduced, and the order quantity of the retail channel should be maintained. Meanwhile product sales prices in both channels should be raised. Consequently, system profit will decrease. The reasons are as follows: Large increase of product sales price in the direct channel and moderate increase of product sales price in the retail channel weaken price advantage of products sold through the direct channel, and cause consumer demand to switch from the direct channel to the retail channel, which results in reduced sales in the direct channel and stable sales in the retail channels.

3) When unforeseen events cause further increase of product price in the retail channel, i.e., $\left(\Delta c_{i}, \Delta c_{d}\right)=(13,3)$, the order quantity in the retail channel should be further reduced, and that in the direct channel should be properly increased. Meanwhile, sales prices in both channels should be raised to deal with the negative influence of unanticipated events. Here, sales price in the retail channel increases more than that in the direct channel, so products sold in the direct channel enjoy more price advantage than that in the retail channel, which results in sales decrease in the retail channel and sales increase in the direct channel. However, product cost increase is more than sales price rise in the retail channel. Therefore, system profit decreases. 
In the same way, as disruptions further increase product cost in the direct channel, i.e., $\left(\Delta c_{i}, \Delta c_{d}\right)=(3,13)$, the order quantity in the direct channel should be substantially reduced and that in the retail channel be slightly raised. Meanwhile product sale prices in both channels are to be raised. The result is the decrease of system profit. This is due to smaller market sizes incurred by increased sales prices in both channels. Here, sales price increases more in the direct channel than in the retail channel, and this leads to price disadvantage in the direct channel, and then causes sales drop in the direct channel. Meanwhile, sales in the retail channel only have limited increase. All these result in reduced system profit.

4) When unforeseen events result in substantial increase of product cost in both channels, i.e., $\left(\Delta c_{i}, \Delta c_{d}\right)=(8,8)$, sales prices of both channels are to be raised. The order quantities of both channel are to be reduced, so is the product output. Consequently, system profit will have a sharp drop. Meanwhile, product cost increases are more than sales price increases, so marginal profits in both channels are substantially reduced. Therefore, in this situation, system profit is 45.583 , and is the minimal profit.

5) When there are relatively small reduction of product costs in both channels, i.e., $\left(\Delta c_{i}, \Delta c_{d}\right)$ $=(-1,-1)$, the optimal output and sales prices are to be maintained as they are in normal situations, but system profit will increase with the reduction of product cost.

6) When product cost reductions in the retail channel are bigger than that in the direct channel, i.e., $\left(\Delta c_{i}, \Delta c_{d}\right)=(-10,-3)$, the order quantity of the retail channel is to be increased, and that in the direct channel is to be maintained. Sales prices of both channels are to be reduced, and system profit will increase. The reasons are obvious. Product cost reductions lead to sales price reduction in both channels, but sales price falls sharply in the retail channel and moderately in the direct channel. This results in bigger market size for the retail channel and stable market size for the direct channel, so the overall system profit substantially increases. Likewise, when the disruptions of unforeseen events lead to bigger product cost reduction in the direct channel, i.e., $\left(\Delta c_{i}, \Delta c_{d}\right)=(-3,-10)$, the order quantity of direct channel is to be increased, and that in the retail channel is to be maintained. Sales prices of both channels should be reduced, and system profit will increase. The reason is that bigger fall of sales price in the direct channel than that in the retail channel further strengthens price advantage of products sold through the direct channel, and expands the market size of the direct channel.

7) When disruptions cause product cost in the retail channel to fall further, i.e., $\left(\Delta c_{i}, \Delta c_{d}\right)=$ $(-13,-3)$, the order quantity of the retail channel should be increased more, and that in the direct channel is to be properly reduced. In the mean time, sales prices of both channels are to be lowered, so as to grab the opportunity brought by unforeseen events, and make more system profit. Here, sales price falls more in the retail channel than in the direct channel, and this greatly expands the market size of the retail channel. Price advantage of the direct channel is weakened, thus sales also fall in the direct channel. But product cost falls more than sales price in the retail channel, so increased output will make more profit for the system, and eventually result in increased system profit. Likewise, when disruptions cause product cost in the direct channel to fall further, i.e., $\left(\Delta c_{i}, \Delta c_{d}\right)=(-3,-13)$, the order quantity of the direct channel should be increased more, and that in the retail channel is to be properly reduced. Sales prices of both channels also need to be lowered, and system profit will increase. The reasons are 
obvious. Lower sales prices in both channels lead to expanded market size. Moreover, sales price falls more in the direct channel than in the retail channel, and strengthens price advantage of the direct channel. This results in considerable sales increase in the direct channel and slight sales decrease in the retail channel. But product cost falls more than sales price in the direct channel, therefore profit of the system will increase.

8) Cost reductions of products sold through both channels are significant, i.e., $\left(\Delta c_{i}, \Delta c_{d}\right)=$ $(-8,-8)$, sales prices of both channels are to be lowered, and order quantities of both channels are to be raised, so is the product output. Consequently, system profit considerably increases.

\section{Summary}

An integrated supply chains with both retail channel and direct channel are discussed in this paper, and adjustment strategies to cope with unanticipated events are provided, based on product cost disruption conditions. The findings are as follows:

1) It is a threat for an integrated supply chain that unanticipated events cause product cost to increase. Increased product cost reduces product market size, and results in impaired system profit. In contrast, it is an opportunity when unanticipated events lead to reduced product cost, since it expands product market size, and increases system profit.

2) Production strategies in normal situations have certain robustness, and should be maintained when product cost disruptions incurred by unanticipated events are relatively small.

3) When unanticipated events cause relatively larger product cost disruptions, sales prices should be adjusted, aligned with the direction that costs are disrupted, i.e., sales prices are raised if product costs increase; sales prices are reduced when product costs decrease. Moreover, the order quantities and the product output should also be properly adjusted as follows:

Unanticipated events may result in increased product cost. When products in one channel undergo greater increase, while those in the other channel undergo smaller increase (disruption is in a certain range), the order quantity in the channel with larger cost increase should be reduced; while in the channel with smaller cost increase, the order quantity should be maintained as in normal situations. As product costs in both channels increase more, the order quantity in the channel with larger cost increase should be reduced more; the order quantity in the channel with smaller cost increase should be increased properly, and the product output should also be reduced.

When product costs of both channels undergo relatively large increase, sales prices are to be raised, and the order quantities of both channels are to be reduced, so is the product output. Here, the impact of unanticipated events on supply chain is relatively great, so system profit will reduced, though adjustment strategies are adopted.

Unanticipated events may also result in reduced product cost. When products in one channel undergo greater decrease, while those in the other channel undergo smaller decrease (disruption is in a certain range), the order quantity in the channel with larger cost reduction should be increased; while in the channel with smaller cost increase, the order quantity should be maintained as in normal situations. As product costs in both channels further decrease, the order quantity in the channel with larger cost decrease should be further increased; the order quantity in the channel with smaller cost decrease should be decreased properly, and the product 
output should also be increased.

When product costs of both channels undergo relatively sharper decrease, the sales prices of both channels are to be reduced, and the order quantities of both channels are to be increased, so is the product output. In this way, opportunity brought by lowered cost can be grabbed to substantially increase system profit.

In this paper, only adjustment strategies to deal with product cost disruptions are put forward. In practice, unanticipated events will also disrupt other factors like market demand, production and operations etc. Adjustment strategies to deal with disruptions of these factors will be studied in future research. Furthermore, interest coordination among integrated supply chain members is also an orientation of our further study in the near future.

Acknowledgements The authors gratefully acknowledge the editor and anonymous referees for their insightful comments and helpful suggestions that led to a marked improvement of the article.

\section{References}

[1] Hendricks K B, Singhal V R. The effect of supply chain glitches on shareholder wealth. Journal of Operation Management, 2003, 21(5): 501-523.

[2] Hendricks K B, Singhal V R. An empirical analysis of the effect of supply chain disruptions on long-run stock price performance and equity risk of the firm. Production and Operations Management, 2005, 14(1): $35-52$.

[3] Causen J, Hansen J, Larsen J. Disruption management. OR/MS Today, 2001, 28(5): 40-43.

[4] Yu G, Qi X T. Disruption management: Framework, models and applications. World Scientific Publishing Co. Pte. Ltd, 2004.

[5] Xanthopoulos A, Vlachos D, Iakovou E. Optimal newsvendor policies for dual-sourcing supply chains: A disruption risk management framework. Computers \& Operations Research, 2012, 39: 350-357.

[6] Huang S, Yang C, Liu H. Pricing and production decisions in a dual-channel supply chain when production costs are disrupted. Economic Modelling, 2013, 30: 521-538.

[7] Xu M, Qi X, Yu G, et al. The demand disruption management problem for a supply chain system with nonlinear demand function. Journal of Systems Science and Systems Engineering, 2003, 12(1): 82-97.

[8] Qi X T, Bard J, Yu G. Supply chain coordination with demand disruptions. Omega, 2004, 32(4): 301-312.

[9] Xiao T, Yu G, Sheng I. Coordination of a supply chain with one-manufacturer and two-retailers under demand promotion and disruption management decisions. Annals of Operations Research, 2005, 135(1): 87-109.

[10] Chen K B, Xiao T J. Demand disruption and coordination of the supply chain with a dominant retailer. European Journal of Operational Research, 2009, 197(1): 225-234.

[11] Huang S, Yang C, Zhang X. Pricing and production decisions in dual-channel supply chains with demand disruptions. Computers \& Industrial Engineering, 2012, 62: 70-83.

[12] Xu M, Qi X T, Yu G. Dyadic supply chains when production costs are disrupted. IIE Transactions, 2006, 38(9): $765-775$.

[13] Li J, Wang S Y, Cheng T C E. Competition and cooperation in a single-retailer two-supplier supply chain with supply disruption. International Journal Production Economics, 2006, 124: 137-150.

[14] Wilson M C. The impact of transportation disruptions on supply chain performance. Transportation Research Part E, 2007, 43: 295-320.

[15] Xiao T J, Qi X T. Price competition, cost and demand disruptions and coordination of a supply chain with one manufacturer and two competing retailers. Omega, 2008, 36(5): 741-753.

[16] Davarzani H, Zegordi S H, Norrman A. Contingent management of supply chain disruption: Effects of dual or triple sourcing. Scientia Iranica E, 2011, 18(6): 1517-1528.

[17] Hallikas J, Virolainenb V M, Tuominen M. Risk analysis and assessment in network environments: A dyadic case study. International Journal of Production Economics, 2002, 78(1): 45-55. 
[18] Tomlin B. On the value of mitigation and contingency strategies for managing supply chain disruption risks. Management Science, 2006, 52(5): 639-657.

[19] Schmitt A J. Strategies for customer service level protection under multi-echelon supply chain disruption risk. Transportation Research (Part B), 2011, 45: 1266-1283.

[20] Craighead C W, Blackhurst J, Rungtusanatham M J, et al. The severity of supply chain disruptions: Design characteristics and mitigation capabilities. Decision Sciences, 2007, 38(1): 131-156.

[21] Dowty R A, Wallace W A. Implications of organizational culture for supply chain disruption and restoration. International Journal Production Economics, 2010, 126: 57-65.

[22] Zegordi S H, Davarzani H. Developing a supply chain disruption analysis model: Application of colored Petri-nets. Expert Systems with Applications, 2012, 39: 2102-2111.

[23] Cachon G P. Supply chain coordination with contracts. Handbooks in Operations Research and Management Science, Supply Chain Management: Design, Coordination and Operation, 2003, 11: 229-339.

[24] Atalay A, Miklos L V. Remanufacturing as a marketing strategy. Management Science, 2008, 54(10): 17311746.

[25] Ferrer G, Swaminathan J M. Managing new and differentiated remanufactured products. European Journal of Operational Research, 2010, 203(2): 370-379. 